08

\title{
Изменение формы наноостровка при селективной эпитаксии
}

\author{
(С) В.Г. Дубровский \\ Санкт-Петербургский государственный университет, Санкт-Петербург, Россия \\ E-mail: dubrovskii@mail.ioffe.ru
}

Поступило в Редакцию 6 апреля 2021 г.

В окончательной редакции 23 апреля 2021 г.

Принято к публикации 23 апреля 2021 г.

\begin{abstract}
Предложена модель, описывающая изменение формы наноостровков, выращиваемых методом селективной эпитаксии. Модель основана на минимизации поверхностной энергии при заданном объеме. Рассмотрена морфология, в которой островок огранен боковыми фасетками (101) и (112) и фасеткой (001) на вершине, размер которых изменяется в зависимости от объема. Проведены расчеты длин, аспектных соотношений и площадей поверхности конкурирующих фасеток в зависимости от объема островка.
\end{abstract}

Ключевые слова: селективная эпитаксия, форма островка, поверхностная энергия.

DOI: 10.21883/PJTF.2021.14.51187.18811

Метод селективной эпитаксии (СЭ) используется для роста полупроводниковых нитевидных нанокристаллов [1-5], наномембран [6-8] и наноостровков $[9,10]$. Метод позволяет контролировать положение наноструктур на поверхности подложки, а также выращивать структуры заранее заданной геометрии. Во многих случаях наноструктуры, выращенные методом СЭ, имеют меньшую плотность структурных дефектов в сравнении с планарными слоями. Это относится, в частности, к наноостровкам фосфида цинка $\left(\mathrm{Zn}_{3} \mathrm{P}_{2}\right)$ - перспективного материала для приложений в фотовольтаике [11-13]. Наноостровки $\mathrm{Zn}_{3} \mathrm{P}_{2}$, выращенные в отверстиях в слое $\mathrm{SiO}_{2}$ на поверхности $\operatorname{InP}(001)$ [10], обладают рядом преимуществ в сравнении с тонкими пленками на различных подложках $[14,15]$. Настоящая работа посвящена моделированию формы наноостровков при наличии конкуренции между различными кристаллографическими гранями, подобной описанной в работе для островков $\mathrm{Zn}_{3} \mathrm{P}_{2}[10]$.

Рассматриваемая геометрия изображена на рис. 1. Островок выращивается в отверстии радиуса $R_{0}$ в оксидной маске на поверхности (001) и представляет собой комбинацию двух усеченных пирамид с известными углами $\alpha_{1}$ и $\alpha_{2}$ при основании. Для пирамиды в виде конуса (это не сказывается на конечных результатах) геометрия островка определяется тремя независимыми параметрами: радиусом основания нижнего конуса $R$, радиусом вершины нижнего конуса $R_{1}$ и радиусом вершины верхнего конуса $R_{2}$. Для определенности будем считать, что боковые фасетки представляют собой низкоэнергетические грани (101) (для нижнего конуса) и (112) (для верхнего конуса), тогда $\alpha_{1}=54.73^{\circ}$ и $\alpha_{2}=45^{\circ}$. Для $v=3 V /\left(\pi \tan \alpha_{1}\right)$ (где $V$ - объем островка над заполненным отверстием) имеем

$$
v=R^{3}-(1-\beta) R_{1}^{3}-\beta R_{2}^{3}, \quad \beta=\tan \alpha_{2} / \tan \alpha_{1}=0.707 .
$$

Нашей целью является описание эволюции системы, изображенной на рис. 1, где происходит переход от усеченной пирамиды, ограненной поверхностью (001) сверху и гранями (112) сбоку, к пирамиде, ограненной фасетками (101). В начале роста имеем плоскую структуру с $R_{1}=R_{2}=R=R_{0}$. Из геометрических соображений $R \geqslant R_{1} \geqslant R_{2}$. Фасетки (112) исчезают при $R_{2}=R_{1}$, тогда как верхняя грань (001) исчезает при $R_{1}=0$. Идея состоит в минимизации поверхностной энергии и

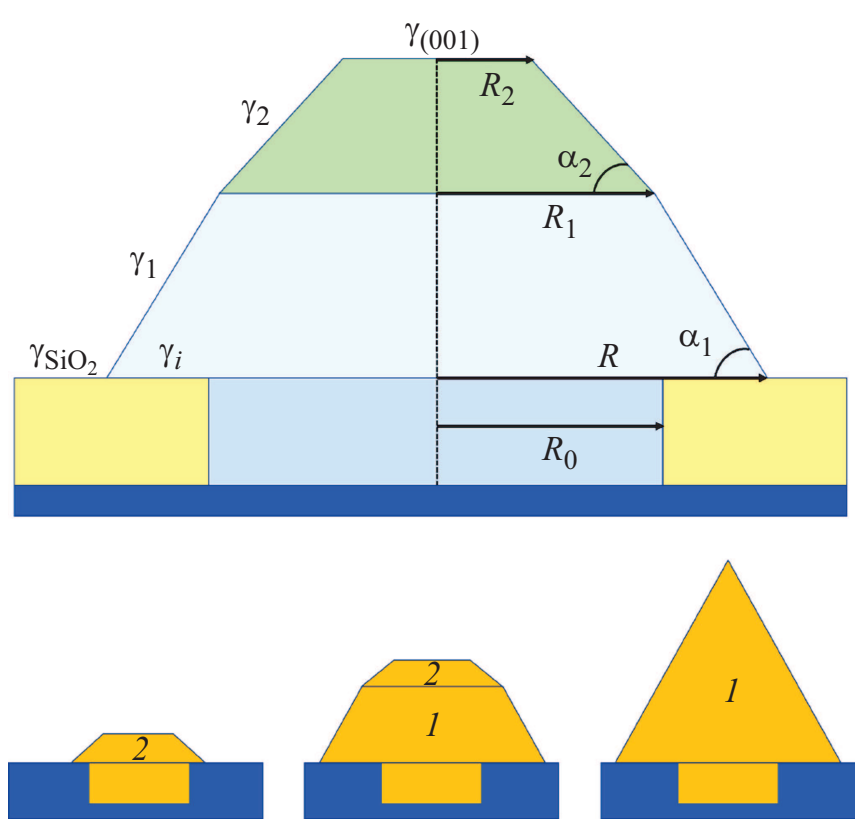

Рис. 1. Модельная геометрия островка. Материал заполняет цилиндрическое отверстие в оксидной маске радиусом $R_{0}$ и затем приобретает трехмерную форму, состоящую из комбинации двух усеченных конусов ( 1 и 2 ). Геометрия определяется тремя радиусами $R, R_{1}$ и $R_{2}$ при известных углах $\alpha_{1}=54.73^{\circ}$ и $\alpha_{2}=45^{\circ}$. Представляющие интерес поверхностные энергии включают энергию горизонтальной плоскости $(001) \gamma_{(001)}$, боковых фасеток (101) $\left(\gamma_{1}\right)$ и (112) $\left(\gamma_{2}\right)$, аморфного слоя $\mathrm{SiO}_{2} \gamma_{\mathrm{SiO}_{2}}$ и интерфейса полупроводниковый материал- $\mathrm{SiO}_{2} \gamma_{i}$. Внизу показано изменение формы островка при увеличении его объема. 
нахождении энергетически выгодной формы островка в зависимости от объема (подобно работам $[4,16]$ ).

Изменение поверхностной энергии при образовании островка равно

$$
\begin{aligned}
& \Delta F_{\text {surf }}=-\pi R_{0}^{2} \gamma_{(001)}+\pi\left(R^{2}-R_{0}^{2}\right)\left(\gamma_{i}-\gamma_{\mathrm{SiO}_{2}}\right) \\
& +\pi\left(R^{2}-R_{1}^{2}\right) \frac{\gamma_{1}}{\cos \alpha_{1}}+\pi\left(R_{1}^{2}-R_{2}^{2}\right) \frac{\gamma_{2}}{\cos \alpha_{2}}+\pi R_{2}^{2} \gamma_{(001)}
\end{aligned}
$$

Первый член - поверхностная энергия зарощенной грани (001) $\mathrm{Zn}_{3} \mathrm{P}_{2}$ в отверстии, второй - изменение поверхностной энергии при формировании интерфейса $\mathrm{SiO}_{2}-\mathrm{Zn}_{3} \mathrm{P}_{2}$ и удалении той же площади поверхности $\mathrm{SiO}_{2}$, третий - энергия боковых граней (101), четвертый - энергия боковых граней (112), пятый - энергия верхней грани (001) (рис. 1). Опустив члены с $R_{0}$, поделив $\Delta F_{\text {surf }}$ на $\pi \gamma_{(001)}$ и выразив $R$ через $R_{1}, R_{2}$ и $v$ из формулы (1) для объема, получаем

$$
\begin{aligned}
f & =(a+b)\left[v+(1-\beta) R_{1}^{3}-\beta R_{2}^{3}\right]^{2 / 3} \\
& +(c-b) R_{1}^{2}-(c-1) R_{2}^{2}
\end{aligned}
$$

с коэффициентами

$$
\begin{gathered}
a=\left(\gamma_{i}-\gamma_{\mathrm{SiO}_{2}}\right) / \gamma_{(001)}, \\
b=\gamma_{1} / \gamma_{(001)} \cos \alpha_{1}, \\
c=\gamma_{2} / \gamma_{(001)} \cos \alpha_{2} .
\end{gathered}
$$

В соответствии с расчетами [10] поверхностные энергии $\mathrm{Zn}_{3} \mathrm{P}_{2}$ равны $\gamma_{1}=\gamma_{(101)}=0.60 \mathrm{~J} / \mathrm{m}^{2}$, $\gamma_{2}=\gamma_{(112)}=0.84 \mathrm{~J} / \mathrm{m}^{2}, \quad \gamma_{(001)}=1.03 \mathrm{~J} / \mathrm{m}^{2}, \quad$ что дает $b=1.01$ и $c=1.15$. Для данной системы в выражении (3) известны все коэффициенты, кроме $a$ (поскольку неизвестна энергия интерфейса $\gamma_{i}$. . Естественно считать $\gamma_{i}<\gamma_{\mathrm{SiO}_{2}}$, поэтому $a<0$. Неравенства $c-b>0$ и $c-1>0$ чрезвычайно важны в дальнейшем. Диффузионный обмен материалом между гранями должен быть достаточно быстрым для осуществления перехода в энергетически выгодную конфигурацию $[17,18]$. Релаксация упругих напряжений при росте на рассогласованных подложках (параметр рассогласования между $\mathrm{Zn}_{3} \mathrm{P}_{2}$ и InP равен 2\% [10]) не учитывается, поскольку такая релаксация должна происходить внутри отверстия [19,20].

Частные производные $f$ по переменным $R_{1}$ и $R_{2}$ равны

$$
\begin{aligned}
\frac{\partial f}{\partial R_{1}} & =\frac{2(a+b)(1-\beta)}{\left[v+(1-\beta) R_{1}^{3}-\beta R_{2}^{3}\right]^{1 / 3}} R_{1}^{2}+2(c-b) R_{1}>0 \\
\frac{\partial f}{\partial R_{2}} & =\frac{2(a+b) \beta}{\left[v+(1-\beta) R_{1}^{3}-\beta R_{2}^{3}\right]^{1 / 3}} R_{2}^{2}-2(c-1) R_{2}
\end{aligned}
$$

Минимум поверхностной энергии по переменной $R_{1}$ достигается при $R_{1}=0$, что соответствует исчезновению верхней грани. Как функция $R_{2}$ поверхностная энергия $f$ имеет минимум при $R_{2}=R_{2 *}$, соответствующем нулю производной (5). Это оптимальное значение зависит от объема $v$ и $R_{1}$ согласно

$$
v=\varepsilon R_{2 *}^{3}-(1-\beta) R_{1}^{3}, \quad \varepsilon=\left[\frac{(a+b) \beta}{c-1}\right]^{3}-\beta .
$$

Таким образом, имеем два уравнения (1) и (6), связывающие три неизвестные величины: $R, R_{1}$ и $R_{2}$. Связь (1) является геометрической и удовлетворяет начальному условию $R_{1}=R_{2}=R=R_{0}$ при $v=0$. Уравнение $(6)$ отвечает минимуму энергии и начальному условию не удовлетворяет. Это означает, что энергетически выгодное значение $R_{2}=R_{2 *}$ устанавливается по достижении некоторого объема. Нахождение трех неизвестных $R$, $R_{1}$ и $R_{2}$ на основании энергетических соображений невозможно. Мы можем, однако, описать эволюцию формы островка в безразмерных переменных

$$
z=\frac{V}{V_{\max }}, V_{\max }=\frac{\pi \tan \alpha_{1}}{3} R^{3}, x=\frac{R_{1}}{R}, y=\frac{R_{2}}{R} .
$$

Очевидно, $V_{\max }$ есть максимальный объем островка в виде полной пирамиды с основанием $R$, поэтому $0 \leqslant z \leqslant 1$. Уравнения (1) и (6) принимают вид

$$
z=1-(1-\beta) x^{3}-\beta y^{3}, \quad z=\varepsilon y_{*}^{3}-(1-\beta) x^{3} .
$$

Теперь можно построить следующую последовательность стадий роста островка.

Стадия 1 (геометрическая)

$$
x=1, \quad y=\left(1-\frac{z}{\beta}\right)^{1 / 3}, \quad 0 \leqslant z \leqslant z_{*} .
$$

Трехмерный островок растет, увеличивая длину боковых фасеток (112) в отсутствие (101). Стадия 1 заканчивается при достижении $z_{*}$, при котором $y\left(z_{*}\right)=y_{*}\left(z_{*}\right)$ : $z_{*}=\beta[\varepsilon-(1-\beta)] /(\beta+\varepsilon)$. Интересная ситуация с конкуренцией различных фасеток требует $0<z_{*}<1$, что накладывает определенные ограничения на значения $a$.

Стадия 2 (энергетическая)

$$
\begin{gathered}
x=\left[\frac{1}{1-\beta}\left(\frac{\varepsilon}{\beta+\varepsilon}-z\right)\right]^{1 / 3}, \\
y=y_{*}=\frac{1}{(\beta+\varepsilon)^{1 / 3}}, \quad z_{*} \leqslant z \leqslant z_{1} .
\end{gathered}
$$

На данной стадии образуются боковые грани (101), что соответствует убыванию $x(z)$. Значение $y$ постоянно и равно энергетически выгодному $y_{*}$. Стадия 2 заканчивается при достижении $z_{1}=z_{*} / \beta$, где $x\left(z_{1}\right)=y_{*}$ и боковые грани (112) полностью исчезают.

Стадия 3 (переход от усеченной к полной пирамиде)

$$
x=(1-z)^{1 / 3}, \quad y=x .
$$

На этой стадии увеличение объема происходит за счет увеличения длины боковых фасеток (101), которые растут до полной пирамиды $(x=0$ при $z=1)$. 
На рис. 2 приведены зависимости $x$ и $y$ от $z$ при $\varepsilon=0.485$ и 3, а также аспектные соотношения $H_{1} / R=\tan \alpha_{1}(1-x) \quad$ и $H_{2} / R=\tan \alpha_{2}(x-y)$, где $H_{1}$ и $H_{2}$ - высоты соответствующих сегментов. При $\varepsilon=0.485$ фасетки (112) имеют малую длину и рано исчезают, после чего островок растет за счет увеличения фасеток (101). При увеличении $\varepsilon$ до 3 фасетки (112) достигают значительной длины и сосуществуют с фасетками (101) длительное время. На рис. 3, $a$ приведены зависимости полной площади поверхности островка $S_{t o t}$ (в единицах $\pi R^{2} / \cos \alpha_{1}$ ) от объема, определяемые выражением $S_{t o t} \cos \alpha_{1} / \pi R^{2}=1-x^{2}+\delta\left(x^{2}-y^{2}\right)+\cos \alpha_{1} y^{2}$, где $\delta=\cos \alpha_{1} / \cos \alpha_{2}=0.812$. На рис. $3, b$ представлены удельные доли площадей поверхности фасеток (001) и (112). При $\varepsilon=0.485$ фасетки (112) быстро исчезают, как и на рис. 2. При $\varepsilon=3$ фасетки (112) ограничивают островок вплоть до объема $z_{*}=0.532$ и исчезают только при $z_{1}=0.732$. Четыре типа морфологии (усеченная пирамида с гранями (112), усеченная пирамида с комбинированными гранями (112) и (101), усеченная пирамида с гранями (101) и полная пирамида с гранями (101)) присутствуют в течение длительного времени.
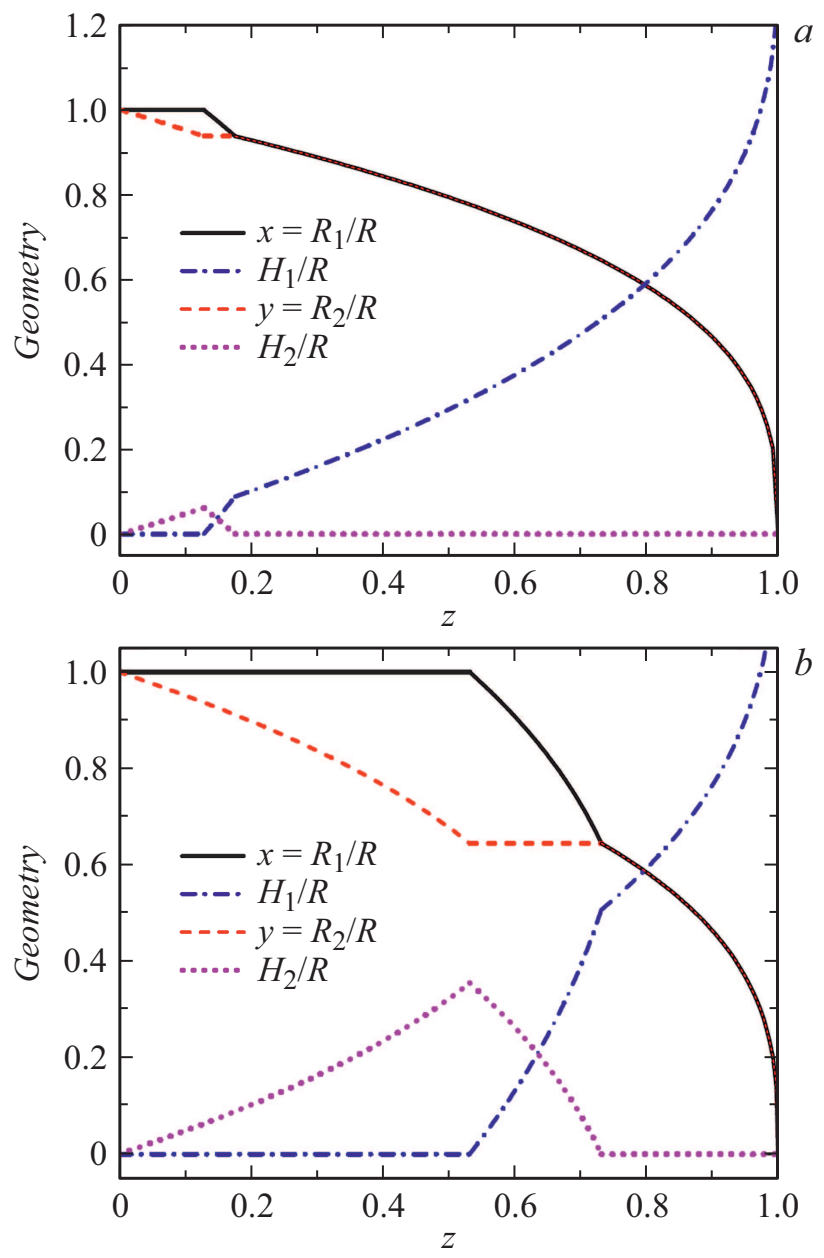

Рис. 2. Зависимости $x, H_{1} / R$ и $y, H_{2} / R$ от $z$ при $\varepsilon=0.485(a)$ и $3(b)$.
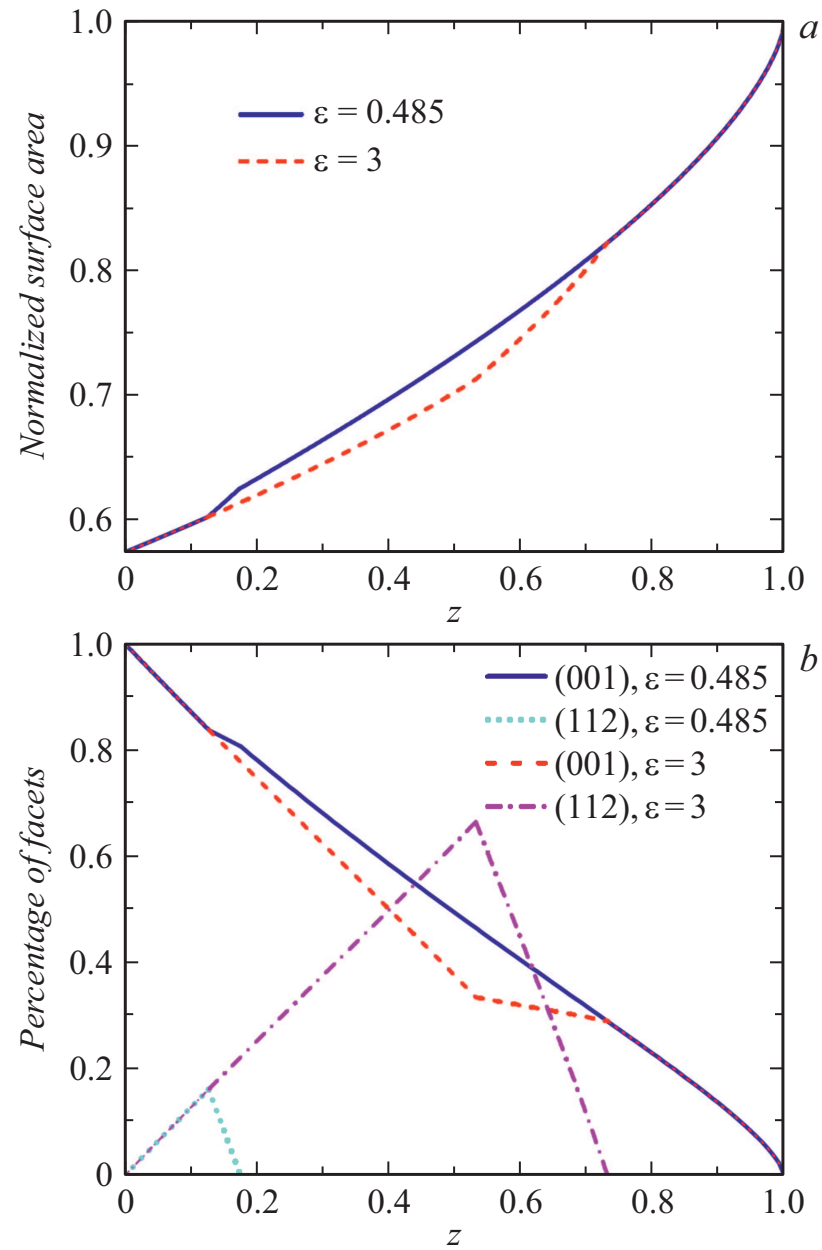

Рис. 3. $a-$ безразмерная площадь поверхности островка $S_{t o t} \cos \alpha_{1} / \pi R^{2}$ в зависимости от $z$ при $\varepsilon=0.485$ и $3 ; b-$ доли площади поверхности фасеток (001) и (112) в зависимости от $z$ при $\varepsilon=0.485$ и 3 .

В работе построена энергетическая модель роста островка в методе СЭ и дана классификация морфологии в зависимости от объема островка, качественно отвечающая росту островков $\mathrm{Zn}_{3} \mathrm{P}_{2}$. Предложенная модель и ее обобщения могут применяться для моделирования и управления формой островков при СЭ для широкого круга материалов.

\section{Финансирование работы}

Работа выполнена за счет гранта Российского научного фонда № 19-72-30004.

\section{Конфликт интересов}

Автор заявляет, что у него нет конфликта интересов. 


\section{Список литературы}

[1] J. Noborisaka, J. Motohisa, T. Fukui, Appl. Phys. Lett., 86, 213102 (2005). DOI: 10.1063/1.1935038

[2] H. Sekiguchi, K. Kishino, A. Kikuchi, Appl. Phys. Lett., 96, 231104 (2010). DOI: 10.1063/1.3443734

[3] Q. Gao, V.G. Dubrovskii, P. Caroff, J. Wong-Leung, L. Li, Y. Guo, L. Fu, H.H. Tan, C. Jagadish, Nano Lett., 16, 4361 (2016). DOI: 10.1021/acs.nanolett.6b01461

[4] J. Vukajlovic-Plestina, W. Kim, L. Ghisalberti, G. Varnavides, G. Tütüncuoglu, H. Potts, M. Friedl, L. Güniat, W.C. Carter, V.G. Dubrovskii, A. Fontcuberta i Morral, Nature Commun., 10, 869 (2019).

DOI: $10.1038 / \mathrm{s} 41467-019-08807-9$

[5] W. Kim, V.G. Dubrovskii, J. Vukajlovic-Plestina, G. Tütncüoglu, L. Francaviglia, L. Güniat, H. Potts, M. Friedl, J.P. Leran, A. Fontcuberta i Morral, Nano Lett., 18, 49 (2018). DOI: 10.1021/acs.nanolett.7b03126

[6] C.-Y. Chi, C.-C. Chang, S. Hu, T.-W. Yeh, S.B. Cronin, P.D. Dapkus, Nano Lett., 13, 2506 (2013).

DOI: $10.1021 / \mathrm{n} 1400561 \mathrm{j}$

[7] M. Bollani, A. Fedorov, M. Albani, S. Bietti, R. Bergamaschini, F. Montalenti, A. Ballabio, L. Miglio, S. Sanguinetti, Crystals, 10, 57 (2020). DOI: $10.3390 /$ cryst 10020057

[8] P. Aseev, A. Fursina, F. Boekhout, F. Krizek, J.E. Sestoft, F. Borsoi, S. Heedt, G. Wang, L. Binci, S. Martí-Sánchez, T. Swoboda, R. Koops, E. Uccelli, J. Arbiol, P. Krogstrup, L.P. Kouwenhoven, P. Caroff, Nano Lett., 19, 218 (2019). DOI: 10.1021/acs.nanolett.8b03733

[9] S. Mokkapati, P. Lever, H.H. Tan, C. Jagadish, K.E. McBean, M.R. Phillips, Appl. Phys. Lett., 86, 113102 (2005). DOI: $10.1063 / 1.1875745$

[10] S. Escobar Steinvall, E.Z. Stutz, R. Paul, M. Zamani, N.Y. Dzade, V. Piazza, M. Friedl, V. de Mestral, J.-P. Leran, R.R. Zamani, A. Fontcuberta i Morral, Nanoscale Adv., 3, 326 (2021). DOI: 10.1039/D0NA00841A

[11] G.M. Kimball, A.M. Mueller, N.S. Lewis, H.A. Atwater, Appl. Phys. Lett., 95, 112103 (2009). DOI: 10.1063/1.3225151

[12] M. Bhushan, A. Catalano, Appl. Phys. Lett., 38, 39 (1981). DOI: $10.1063 / 1.92124$

[13] T. Suda, K. Kakishita, J. Appl. Phys., 71, 3039 (1992). DOI: $10.1063 / 1.350989$

[14] J.P. Bosco, G.M. Kimball, N.S. Lewis, H.A. Atwater, J. Cryst. Growth., 363, 205 (2013). DOI: $10.1016 /$ j.jcrysgro.2012.10.054

[15] R. Paul, N. Humblot, S. Escobar Steinvall, E.Z. Stutz, S.S. Joglekar, J.-B. Leran, M. Zamani, C. Cayron, R. Logé, A.G. del Aguila, Q. Xiong, A. Fontcuberta i Morral, Cryst. Growth Design, 20, 3816 (2020). DOI: 10.1021/acs.cgd.0c00125

[16] M. Zeghouane, Y. André, G. Avit, J. Jridi, C. Bougerol, P.-M. Coulon, P. Ferret, D. Castelluci, E. Gil, P. Shields, V.G. Dubrovskii, A. Trassoudaine, Nano Futures, 4, 025002 (2020). DOI: 10.1088/2399-1984/ab8450

[17] V.G. Dubrovskii, Phys. Status Solidi B, 171, 345 (1992). DOI: $10.1002 / p s s b .2221710206$

[18] M. Albani, R. Bergamaschini, M. Salvalaglio, A. Voigt, L. Miglio, F. Montalenti, Phys. Status Solidi B, 256, 1800518 (2019). DOI: $10.1002 /$ pssb.201800518

[19] F. Glas, Phys. Rev. B, 74, 121302(R) (2006). DOI: 10.1103/PhysRevB.74.121302

[20] V.G. Dubrovskii, N.V. Sibirev, X. Zhang, R.A. Suris, Cryst. Growth Design, 10, 3949 (2010). DOI: 10.1021/cg100495b 